\title{
Design and Analysis of a Low Power UWB Pulse Generators
}

\author{
Saleh M. Eisa, Hanady H. Issa, Khaled A. Shehata, and Hani F. Ragai
}

\begin{abstract}
This paper presents three configurations of Ultra Wideband (UWB) Pulse Generators (PGs). The Generators target low power applications operating in the frequency range 3.1-10.6 GHz. The designs use logic gates and buffered filters to generate a Gaussian 5th derivative pulse. The used technology is $0.13 \mu \mathrm{m}$ from TSMC. The lowest power consumption and highest $B W$ achieved are $0.13 \mathrm{~mW}$ and $7.1 \mathrm{GHz}$ respectively. In terms of Power Spectral Density (PSD), the designs comply with the $-41.3 \mathrm{dBm}$ FCC requirements.
\end{abstract}

Index Terms-Ultra wideband, pulse generators, gaussian pulse, ring oscillator.

\section{INTRODUCTION}

Signals having a Bandwidth (BW) of $500 \mathrm{MHz}$ or a Fractional Bandwidth (BWf) less than or equal to 0.2 are considered as UWB signals according to the Federal Communications Commission (FCC) definition [1]. In order for UWB applications to coexist with common wireless communication systems without any interference, the FCC has defined PSD masks for different applications. Indoor UWB applications have an allocated bandwidth that extends from 3.1 to $10.6 \mathrm{GHz}$ with a maximum PSD of $-41.3 \mathrm{dBm}$. This PSD level represents the unintentional power radiated from television sets or monitors. UWB signals are characterized by short pulses with duration less than $1 \mathrm{nsec}$, relatively low power and wide BW. These characteristics migrate UWB from military applications towards commercial applications. Because of its wide BW, UWB signals are suitable for high data rate applications over short distances (several meters) such as wireless Personal Area Networks (WPAN) [2]. UWB can even be used for shorter distance applications such as Body Area Networks (BAN) [3]. Besides, high data rates UWB signals can also be used for low data rate with higher range as in Wireless Sensor Networks (WSN) [4] and Radio Frequency Identification (RFID) applications [5].

\section{UWB PULSE GENERATION}

The Pulse Generator (PG) is the most important part of the UWB system. Design simplicity is the most attractive aspect of UWB PGs when compared to common communication systems. Different pulse shapes such as rectangular, cosine and Gaussian pulses are used for UWB communications.

Manuscript received November 9, 2013; revised March 6, 2014

S. M. Eisa, H. H. Issa, and K. A. Shehata are with the Arab Academy for Science, Technology and Maritime Transport, Cairo, Egypt (e-mail: saleheisa@cairo.aast.edu, Hanady.issa@aast.edu, k_shehata@aast.edu)

H. F. Ragai Issa is with Ain-Shams University, Cairo, Egypt (e-mail: hfragai@ieee.org)
Among all, the Gaussian pulse is the most common used because of its ease in generation. In addition, Gaussian pulse has a smooth transition in time domain and better frequency response. Step Recovery Diodes (SRD) and avalanche transistors are employed to generate Gaussian pulses [6], [7]. Logic gates are also used to generate Gaussian pulses [8]. To comply with the FCC requirements, pulse shaping filters are used to transform the generated pulse to a higher order derivatives Gaussian pulse [9].

This paper proposes three different UWB PGs. The core of the proposed PGs is the ring oscillator. The generated pulse is then shaped to produce a Gaussian 5th derivative pulse that complies with FCC regulations. The shaped pulse has duration less than $1 \mathrm{nsec}$. The generated pulse occupies a frequency band from 3.1 to $10.6 \mathrm{GHz}$. The PGs are designed on TSMC $0.13 \mu \mathrm{m}$ CMOS technology and $1.2 \mathrm{~V}$ voltage supply using Cadence tools.

\section{DESIGN 1: FREE RUNNING 21-STAGE RING OSCILLATOR UWB PG}

The first PG design consists of a 21-stage ring oscillator, gated clock generator, Gaussian pulse generator, buffer circuit and a shaping filter module as shown in Fig. 1.

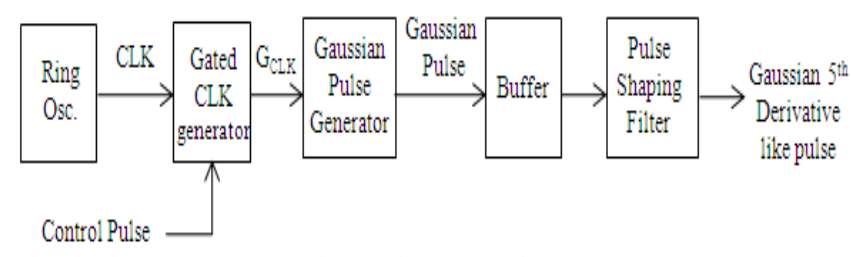

Fig. 1. First PG design.

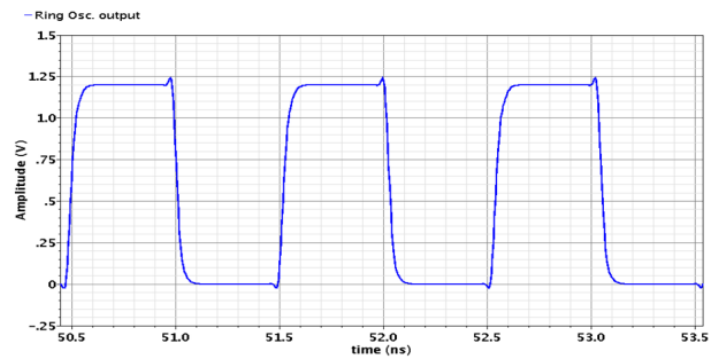

Fig. 2. Ring oscillator output.

The stages used in this oscillator utilize a minimum sized NMOS while the PMOS is designed to compensate the difference in mobility. A series of simulations are executed to determine the rise and fall time for the generated pulse associated with the oscillator. The number of inverter stages needed to produce a $1 \mathrm{nsec}$ output signal is 21 stages. The simulated output of the designed ring oscillator is plotted in 
Fig. 2. An AND gate is used as a gated clock generator. The AND gate inputs are driven by the ring oscillator output and a $20 \mathrm{~ns}$ control pulse. The control pulse is used to adjust the overall bit rate of the system. The Gaussian Pulse is generated from a two-input NOR gate. One input is driven directly from the gated clock and the other is driven by a delayed one as shown in Fig. 3. The generated Gaussian pulse with a 250 psec width is shown in Fig. 4.

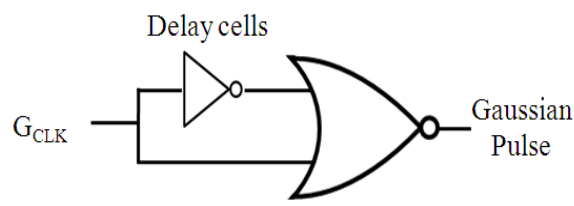

Fig. 3. The Gaussian pulse generator module.

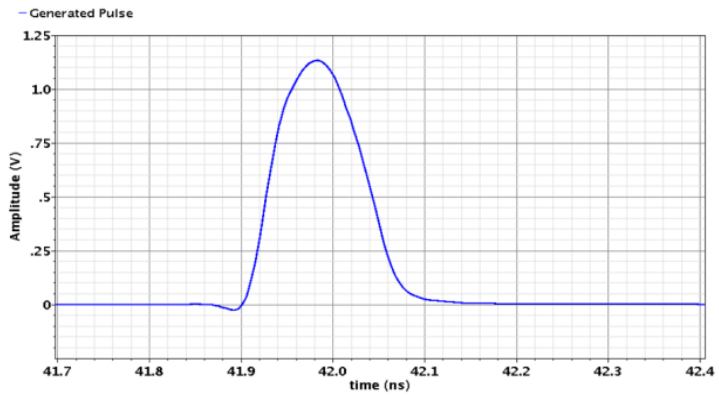

Fig. 4. The generated Gaussian pulse.

The pulse shaping filter in Fig. 1 is constructed using LC pi section 3rd order Butterworth Band Pass Filter (BPF). The filter type is chosen because it has the maximum flat response in its mid band [10]. The $-10 \mathrm{~dB}$ bandwidth of the designed filter is $7.5 \mathrm{GHz}$. ADS tools are used in designing this filter. The equivalent input and output impedances of the filter are $50 \Omega$ to match the buffer output and antenna load impedances. The S12 parameter of the BPF is plotted in Fig. 5. The filter response shows that the lower and upper $-10 \mathrm{~dB}$ cutoff frequency are 3.2 and $13 \mathrm{GHz}$ respectively. Due to components degradation at high frequencies the upper cutoff frequency will comply with the FCC regulations.

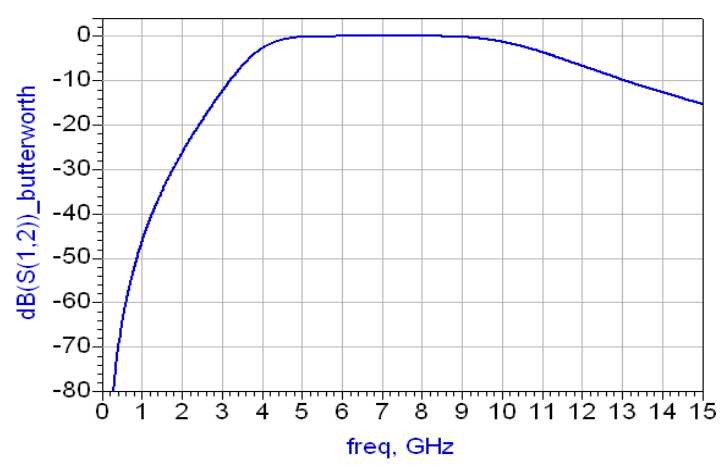

Fig. 5. S12 parameter of the Butterworth filter.

A buffer is utilized to separate the Gaussian Pulse Generator (GPG) circuit from the shaping filter. In addition, it is used to transform and match the GPG and load impedances for better filter response. The buffer consists of 6 stages with a stage ratio of 3 [11]. The first and last stages are designed to match the $11.2 \mathrm{k} \Omega$ output impedance of the GPG with the $50 \Omega$ input impedance of the filter. The generated pulse has $660 \mathrm{mV}$ peak-to-peak amplitude and 590 psec pulse duration as shown in Fig. 6. The pulse BW extends from 3.1 to $10.2 \mathrm{GHZ}$ with a maximum PSD of $-41.5 \mathrm{dBm}$ at $5.4 \mathrm{GHz}$ as shown in Fig. 7.

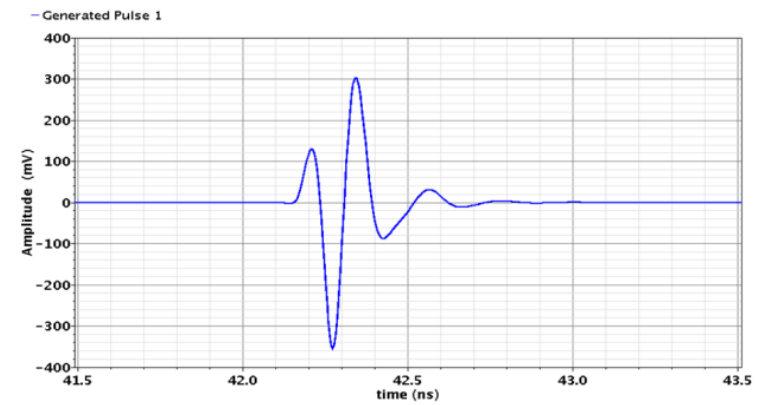

Fig. 6. Pulse generated from design 1 .

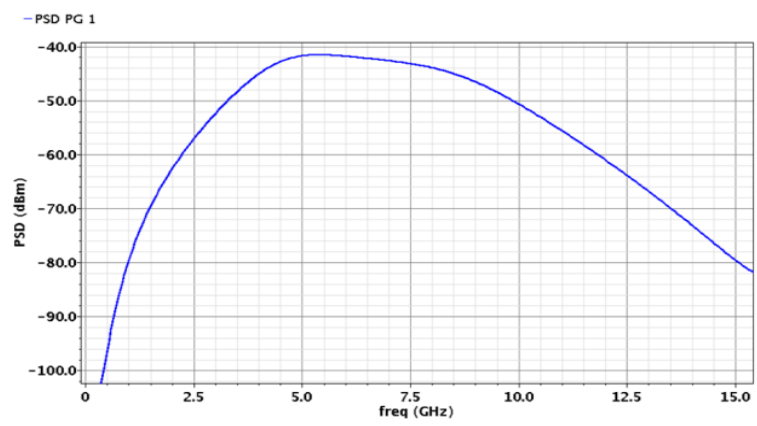

Fig. 7. PSD of generated signal in design 1.

\section{DESIGN 2: A CONTROLLED 21-STAGE Ring OSCILLATOR UWB PG.}

The second PG design consists of a voltage controlled ring oscillator, a Gaussian pulse generator, buffer circuit and a pulse shaping filter as shown in Fig. 8. The controlled 21-stage ring oscillator is based on the same design presented in [9], [12]. An NMOS transistor is connected in series with the other 21 stages as shown in Fig. 9. In Addition, a PMOS transistor is inserted between the first two stages. The output pulse of the controlled oscillator is similar to the gated clock generated in design 1.

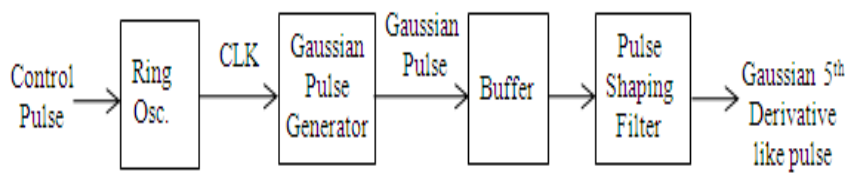

Fig. 8. Second PG design.

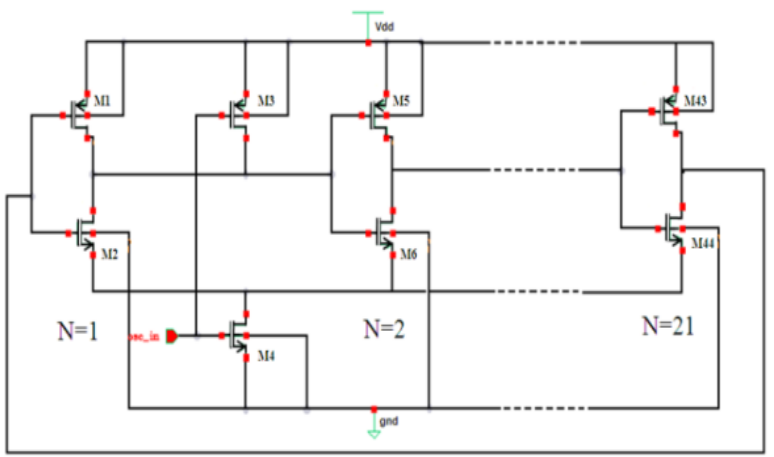

Fig. 9. A 21-Stage controlled ring oscillator.

A control signal is used to turn the added transistors ON/OFF and thus control the current pass to the ground. 
When the control signal is high the added NMOS transistor is turned $\mathrm{ON}$ allowing the oscillator to run. When the control signal is low the output of the oscillator is pulled up to $\mathrm{V}_{\mathrm{DD}}$ by the added PMOS. The same circuit for the Gaussian pulse generator, buffer circuit and shaping filter presented in the first design are utilized. The generated Gaussian 5th derivative pulse is presented in Fig. 10.

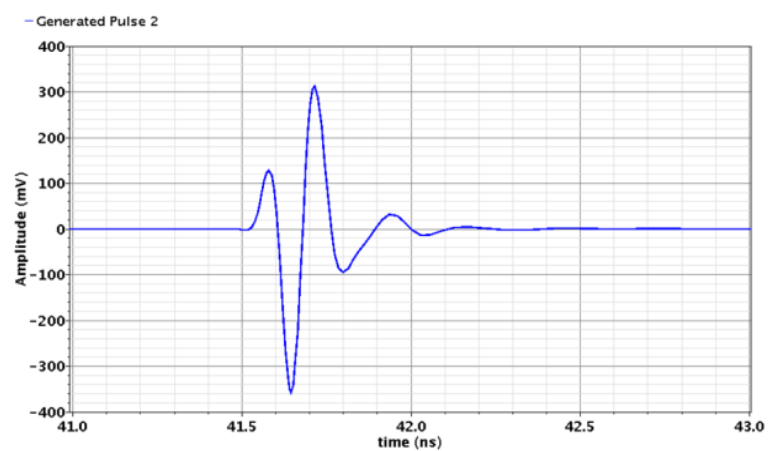

Fig. 10. Pulse generated from design 2.

The generated pulse has peak-to-peak amplitude of 670 $\mathrm{mV}$ and pulse width of 590 psec. The PSD shows that the generated pulse complies with FCC requirements as Fig. 11. The $\mathrm{BW}$ of the pulse is $7 \mathrm{GHz}$ with lower and upper cutoff frequencies 3.1 and $10.1 \mathrm{GHz}$ respectively. The maximum simulated PSD is $-41.3 \mathrm{dBm}$ at $5.3 \mathrm{GHz}$.

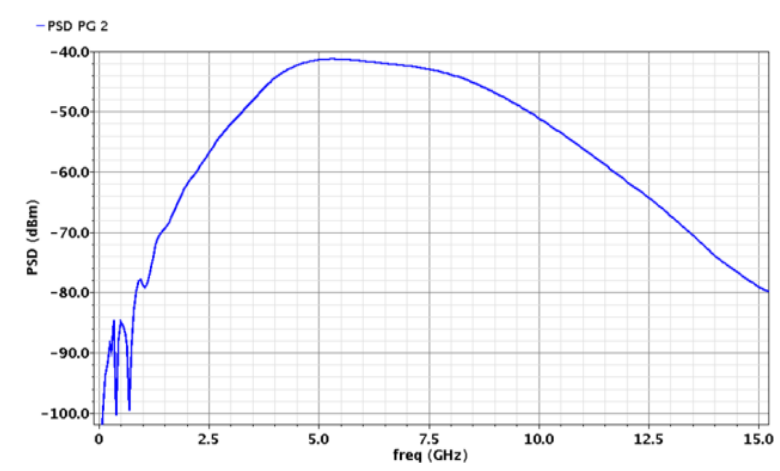

Fig. 11. PSD of generated signal in design 2.

\section{Design 3: A Controlled 3-Stage Ring Oscillator UWB PG}

In this design a 3-stage controlled ring oscillator is used. The design is based on the same idea presented in design 2 [9]. The block diagram for the pulse generator is shown in Fig. 12. The Control pulse switches the oscillator ON and OFF. The oscillator generates Gaussian pulse with 250 psec pulse duration. The peak-to-peak amplitude of the generated pulse is $690 \mathrm{mV}$ with pulse width of $690 \mathrm{psec}$ as shown in Fig. 13. The pulse has a maximum PSD of $-41 \mathrm{dBm}$ at $5.3 \mathrm{GHz}$ as shown in Fig. 14. The BW of the pulse is $6.8 \mathrm{GHz}$ with lower and upper cutoff frequencies of 3.1 and $9.9 \mathrm{GHz}$ respectively.

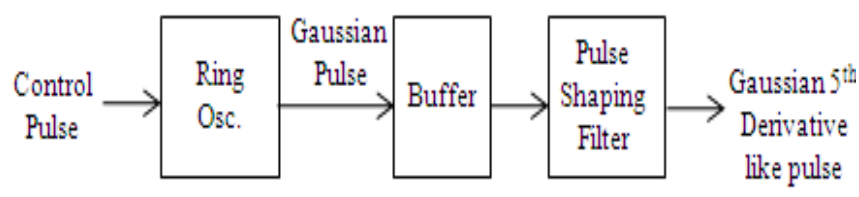

Fig. 12. Third PG design.

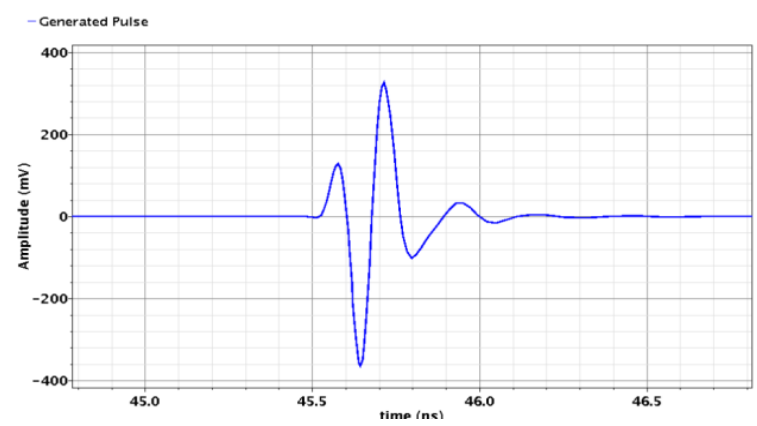

Fig. 13. Pulse generated from design 3

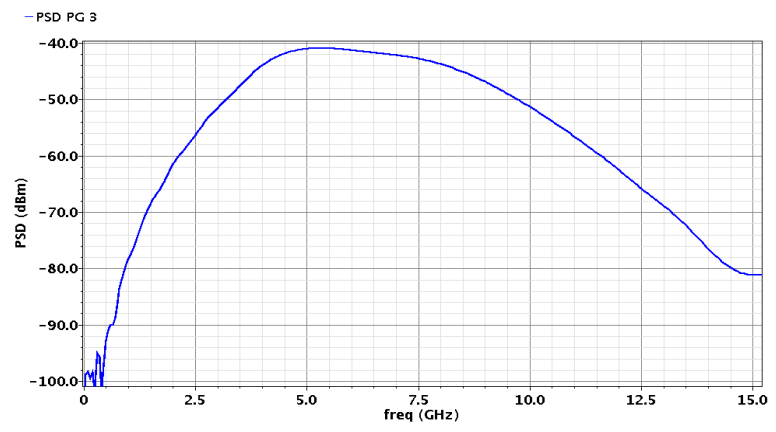

Fig. 14. PSD of generated signal in design 3.

\section{Simulation Results AND Discussion}

The buffering and shaping filter circuits are identical in the three designs. The only difference between the three designs is the method of generating the Gaussian pulse, mainly the ring oscillator. The total power dissipation, peak-to-peak amplitude, BW and the PSD are simulated. Table I shows that the three designs have comparable results in terms of BW and PSD. Design 3 has the highest output voltage while design 1 has the lowest voltage. Design 2 and 3 consume the same power while design 1 consumes $54 \%$ more power. This can be attributed to the free running 21-stage ring oscillator.

\section{TABLE I: COMPARISON AMONG THE THREE DESIGNS}

\begin{tabular}{|c|c|c|c|c|}
\hline \multirow{2}{*}{$\begin{array}{c}\text { Pulse } \\
\text { Generator }\end{array}$} & $\begin{array}{c}\text { Total Pavr. } \\
(\mathrm{mW})\end{array}$ & $\begin{array}{c}\text { Amp. } \\
(\mathrm{mVp}-\mathrm{p} \\
)\end{array}$ & $\begin{array}{c}-10 \mathrm{~dB} \text { BW } \\
(\mathrm{GHz})\end{array}$ & $\begin{array}{c}\text { PSD } \\
(\mathrm{dBm})\end{array}$ \\
\hline \hline Design 1 & 0.2 & 660 & $3.1-10.2$ & -41.5 \\
\hline Design 2 & 0.13 & 670 & $3.1-10.1$ & -41.3 \\
\hline Design 3 & 0.13 & 690 & $3.1-9.9$ & -41 \\
\hline
\end{tabular}

\section{CONCLUSION}

Three designs for UWB PGs are presented in this paper. The designs utilize ring oscillators with different number of stages to generate a Gaussian pulse. The pulse is then transformed to its 5th derivative using a Butterworth BPF filter. The Design containing a free running 21-stages ring oscillator consumes $0.2 \mathrm{~mW}$ which is the highest power consumption among the three designs. While the designs with the controlled ring oscillators consume $0.13 \mathrm{~mW}$. The PSDs of the three designs vary from -41 to $-41.5 \mathrm{dBm}$ which satisfy the FCC requirement for indoor applications. The accomplished BWs range from 6.8 to $7.1 \mathrm{GHz}$ satisfying the 
UWB requirements.

\section{REFERENCES}

[1] Federal Communication Commission: First Report and Order, FCC, pp. 02-48, Feb. 14, 2002.

[2] M. Demirkan and R. R. Spencer, "A pulse-based ultra-wideband transmitter in 90-nm CMOS for WPANs," IEEE Journal of Solid-State Circuits, vol. 43, no. 12, pp. 2820-2828, December 2008.

[3] R. K. Dokania, X. Y. Wang, S. G. Tallur, and A. B. Apsel, "A Low Power Impulse Radio Design for Body-Area-Networks," IEEE Transactions on Circuits and Systems-I: Regular Papers, vol. 58, no. 7, pp. 1458-1469, July 2011.

[4] R. Agieb, I. Adly, and H. Ragai, "Two nodes UWB low power asset localization in WSN," presented at International Conference of Computer Application and Technology, January 2013.

[5] A. Lazaro, A. Ramos, D. Girbau, and R. Villarino, "A novel UWB RFID tag using active frequency selective surface," IEEE Transactions on Antennas and Propagations, vol. 61, no. 3, pp. 1155-1165, March 2013.

[6] H. H. Issa, S. M. Eisa, K. A. Shehata, and H. F. Ragai, "SRD-based pulse generator for UWB wireless network applications," in Proc. International Conference of Computer Application and Technology, January 2013.

[7] Z. Shahid, S. Khan, and Z. Alam, "Pulse generation using avalanche transistor for ultra wide band communication: analysis and simulation results," in Proc. International Conference on Computer and Communication Engineering, July 2012, pp. 625-628.

[8] X. Wang, S. Q. Fan, H. Tang, L. Lin, J. Liu, Q. Fang, H. Zhao, A. Wang, L. W. Yang, and B. Zhao, "A whole-chip ESD-protected 0.14-pJ/p-mV3.1-10.6-GHz impulse-radio UWB transmitter in

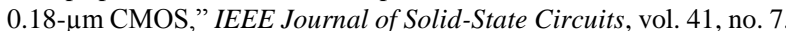
pp. 1109-1115, July 2006.

[9] J. B. Radic, A. M. Djugova, L. F. Nagy, and M. S. Videnovic-Misic, "A low power 3.1-7.5 GHz tunable pulse generator for impulse radio UWB," in Proc. IEEE 10th Jubilee International Symposium on Intelligent Systems and Informatics, September 2012, pp. 425-428.

[10] R. Ludwing and P. Bretchko, RF Circuit Design: Theory and Application, Prentice Hall, 2000, ch. 5 .

[11] N. H. E. Weste and D. M. Harris, CMOS VLSI Design: A Circuits and Systems Perspective, 4th ed. Pearson Education Inc., 2011, ch. 4, pp. 163-173.

[12] B. Qin, X. Wang, H. L. Xie, L. Lin, H. Tang, A. Wang, H. Y. Chen, B. Zhao, L. W. Yang, and Y. M. Zhou, "1.8 pJ/pulse programmable Gaussian pulse generator for full-band noncarrier impulse-UWB transceivers in 90-nm CMOS," IEEE Transactions on Industrial Electronics, vol. 57, no. 5, pp. 1555-1562, May 2010.

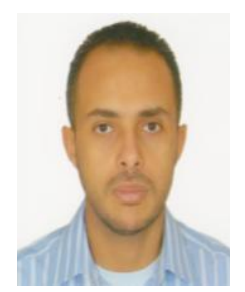

Saleh M. Eisa received his B.Sc. and M.Sc. degrees from the Arab Academy for Science, Technology and Maritime Transport (AASTMT) in 2000 and 2006 respectively. He is currently working towards the Ph.D. degree at Ain Shams University, Cairo, Egypt. Eisa is working as a teaching assistant at the AASTMT, Electronics and Communication Department, Cairo Branch. His research interests include mixed signal design, VHDL based design and electronic system design. His research interests include analog and digital VLSI.

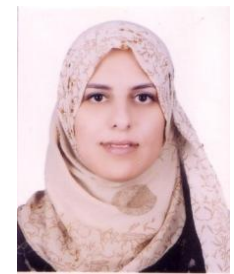

Hanady H. Issa received her B.Sc. from Arab Academy for Science, Technology and Maritime Transport (AASTMT) in 1998. She worked as a teaching assistant in AAST. She received her M.Sc. from AAST Egypt in 2003. She received her Ph.D degree in electronics and communication engineering from Ain Shams University, Egypt, in 2009. She is currently an associate professor at AAST, Electronics and Communication Department, Egypt. Her research interests include mixed signal design, electronic system design, VHDL based FPGA design, simulation and synthesis.

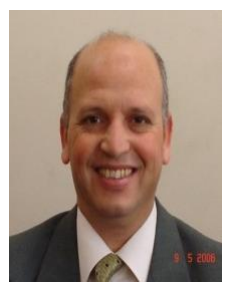

Khaled A. Shehata received his B.Sc. from Military Technical College, Cairo, Egypt in 1981. After working as a research assistant he got his M.Sc. from Cairo University, Egypt in 1991. He received his PhD. from Naval Postgraduate School, Monterey, California, USA in 1996. He worked as a researcher in Egypt, then the director for the VLSI design center, AOI, Egypt, finally he is a professor in the Arab Academy for Science, Technology and Maritime Transport, College of Engineering since 2000 till now. He is the chairman of Electronics and Communication Department. His research interests include analog and digital VLSI design, electronic system design and have more than 80 scientific research papers in these areas.

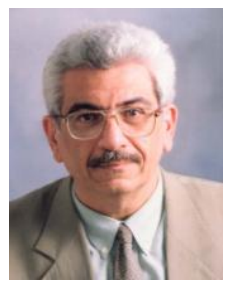

Hani Fikry Ragai received the B.Sc. and M.Sc. degrees from Ain Shams University (ASU), Cairo, Egypt in 1967 and 1972 respectively. He received the "doctorat de specialite" and the "doctorat d'etat" in electronics from Grenoble University and INPG, France in 1976 and 1980 respectively. From 1972 to 1979 he was a research assistant at LETI, CEA and then at THOMSON-EFCIS from 1979 to 1980, both in Grenoble, France. He then joined the Electronics and Communication Engineering (ECE) Department, Faculty of Engineering, Ain Shams University as assistant professor from 1980 to 1985 . Between 1981 and 1984 he was with the solar energy consultancy sector, CEDUST, French Embassy in Cairo. From 1982 and 1984 he joined the solar and photovoltaic joint research project between Alex University and Delft University. From 1985 to 1990 he was with King Saud University, Riyadh, Saudi Arabia. Since 1990 he is a professor of electronics in ECE department, ASU where he was also the director of the "Integrated Circuits Lab" till 2006. Between 1997 and 2002 he was consultant in Mentor Graphics and MEMScAP, Egypt. From 2006 to 2011 he became professor emeritus at Ain Shams University and is also seconded to the French University of Egypt as head of the Information and Communications Technology department. He served as co-chair for the International Conference in Microelectronics, ICM 2007. Since 1981 and till now he co-advised more than $80 \mathrm{M}$.Sc./PhD theses and co-authored more than 160 papers and one book on Electronic Devices. His research interests include modeling and characterization of solar cells and MOS devices, ASICs for wireless sensor nodes, wireless sensor networks for industrial applications, and MEMS-based sensors and energy scavenging. 